Note: this is a peer-reviewed and approved draft of the journal article:

Chanda, S., Dickinson, A.S., Gupta, S., Browne, M., (2015) “Full-field In vitro Measurements and In silico Predictions of Strain Shielding in the Implanted Femur after Total Hip Arthroplasty”. Proceedings of the Institution of Mechanical Engineers Part H: Journal of Engineering in Medicine, IN PRESS, DOI: 10.1177/0954411915591617

This document will be updated when the final, fully proofed article is made available online by the publisher, SAGE Journals. 


\title{
Full-field In vitro Measurements and In silico Predictions of Strain Shielding in the Implanted Femur after Total Hip Arthroplasty
}

Authors and affiliations:
(1) Souptick Chanda ${ }^{1}$
(2) Alexander Dickinson ${ }^{2}$
(3) Sanjay Gupta ${ }^{1}$
(4) Martin Browne

${ }^{1}$ Department of Mechanical Engineering, Indian Institute of Technology Kharagpur, Kharagpur 721 302, West Bengal, India. ${ }^{2}$ Bioengineering Science Research Group, School of Engineering Sciences, University of Southampton, Southampton S017 1BJ, United Kingdom.

Corresponding author:

Dr. Sanjay Gupta

Department of Mechanical Engineering

Indian Institute of Technology Kharagpur

Kharagpur 721 302, West Bengal, India

Tel: $+91-(0) 3222-282958$

Fax: $+91-(0) 3222-282277$

Email: sangupta@ mech.iitkgp.ernet.in

Keywords: Total Hip Arthroplasty, Bone adaptation, Mechanical testing, Digital Image Correlation, Finite element analysis.

\begin{abstract}
Alterations in bone strain as a result of implantation may contribute towards periprosthetic bone density changes after Total Hip Arthroplasty (THA). Computational models provide full-field strain predictions in implant-bone constructs; however, these predictions should be verified using experimental models wherever possible. In the present work, finite element (FE) predictions of surface strains in intact and implanted composite femurs were verified using digital image correlation (DIC). Relationships were sought between post implantation strain states across seven defined Gruen zones (GZ) and clinically observed longer term bone density changes. Computational predictions of strain distributions in intact and implanted femurs were compared to DIC measurements in two regions of interest. Regression analyses indicated strong linear correlation between measurements and predictions $(\mathrm{R}=0.927$ intact, 0.926 implanted) with low standard error $(\mathrm{SE}=38 \mu \varepsilon$ intact, 26 $\mu \varepsilon$ implanted). Pre- to postoperative changes in measured and predicted surface strains were found to relate qualitatively to clinicallyobserved volumetric bone density changes across seven Gruen zones: marked proximal bone density loss corresponded with a 50-64\% drop in surface strain, and slight distal density changes corresponded with 4-14\% strain increase. These results support the use of DIC as a pre-clinical tool for predicting post implantation strain shielding, indicative of long-term bone adaptations.
\end{abstract}


Adverse implant-induced bone adaptation can compromise the longevity of cementless THA. ${ }^{1-3}$ Understanding how bone strain is influenced by implantation may explain in part the mechanical contribution to periprosthetic bone density changes. ${ }^{4,5}$ Several numerical predictions of trabecular bone adaptation have been carried out using FE analysis based on differential strain stimulus in the human femur. ${ }^{6-8}$ However, simplified bone material properties, geometry and loading conditions have been limitations in most of such FE models, since the femur is known to exhibit a complex morphology and heterogeneous material properties. ${ }^{9}$ Experimental testing employing strain gauges offers an alternative method for measuring strain fields for the assessment of post-operative bone response in vitro. ${ }^{10-13}$ However, the restricted area of interrogation associated with strain gauges is considered to be a limitation of the technique and recently, non-destructive optical measurement methods such as Digital Image Correlation (DIC) have gained popularity because of their full-field data-rich strain measurement capability. ${ }^{14-16}$

DIC is a well-established non-contact optical metrology method that extracts full-field deformation measurements of objects subjected to external loads. ${ }^{17,18}$ It is particularly useful for measuring deformations in complex geometries with inhomogeneous material properties, like human bone, by finding spatial correspondences between different digital images. ${ }^{19}$ Use of DIC can further avert reinforcement effects associated with strain gauging, which originate from the stiffness of the sensor mounted onto (or embedded into) the tested material. ${ }^{20-22}$ Recently, a series of investigations has been carried out using DIC to measure strains over bone surfaces. ${ }^{14-16,23-25}$

Despite its strong potential, the use of DIC has largely been restricted to FE validation purposes in experimental biomechanics. Moreover, only a few such investigations were carried out on the femur, either using composite ${ }^{14,19,26,27}$ or cadaver specimens. ${ }^{25,28-30}$ Tayton et al. ${ }^{26}$ employed DIC to find the differences in 2-D strain patterns generated in the medial cortex of composite femurs implanted with femoral prostheses of different material stiffness. Dickinson et al. ${ }^{14}$ tested one composite femur bone in quasi-static loading, and measured the strain distribution using DIC in order to validate a corresponding FE model. DIC data were averaged within 25 different $5 \mathrm{~mm}^{2}$ areas, and compared to equivalent strain magnitude predictions from the FE model. Similar techniques have been applied to pelvises in order to evaluate different acetabular cup materials. ${ }^{31}$ Op Den Buijs and Dragomir-Daescu ${ }^{25}$ obtained DIC measurements from 22 human cadaver femora in order to validate FE models in terms of overall stiffness, and to tune the simulation parameters.

Recent investigations on human cadaver femora have used DIC to study femur fracture. In a study of simulated sideways falling using a drop tower test protocol, multiple human cadaver femora were tested using DIC to investigate femoral neck fracture. ${ }^{28}$ A DIC-FE based experimental-computational technique was adopted to study the influence of local bone structural weaknesses over femur fracture initiation. ${ }^{30}$ Grassi et al. ${ }^{29}$ employed DIC to measure the full-field strain response of three cadaver femora tested at a physiological strain rate up to fracture under a single-leg stance configuration.

Whilst several studies have employed full-field experimental strain measurements to validate computational predictions and to verify bone constitutive laws and fracture criteria, there is a dearth of full-field experimental strain studies on intact and implanted femurs, to support computational predictions of implant-induced long-term bone response. The primary objective of this study was to measure surface strains in intact and implanted composite femurs using DIC in order to (1) validate the full-field numerical strains predicted by the corresponding FE models and to (2) quantitatively assess the implant-induced surface strain changes across seven defined Gruen zones. Furthermore, an attempt was made to verify whether clinically observed bone density changes can be qualitatively related to the DIC measured and FE predicted surface strain changes. 


\section{Materials and Methods}

One large sized Sawbone femur (model 3406) was prepared and implanted with a Furlong HAC (hydroxyapatitecoated) stem of size $12 \mathrm{~mm}$ by an experienced orthopaedic registrar. A high contrast, random speckle pattern was applied on the surface of both femurs for DIC measurements: matt white undercoat was first applied, followed by black speckles. This was done by dipping a toothbrush in water soluble acrylic black paint and then rubbing the thumb over the surface of the bristles to flick the speckles on the specimen.

\section{DIC experimental set-up and measurements}

The femur specimens were potted one-third along the shaft from the distal end and tilted laterally by $\sim 10^{\circ}$ in the frontal plane and by $\sim 10^{\circ}$ dorsally in the sagittal plane (Fig. 1 a,b). This orientation is similar to that described for fatigue testing of hip prostheses (ISO 7206 - 4:2002 Implants for surgery), designed to represent typical gait load at heel strike. ${ }^{32}$ The potted assembly was fixed to the base of an Instron testing machine (Instron5569, Instron Ltd., High Wycombe, UK). A vertical downward force was applied through a $28 \mathrm{~mm}$ diameter modular femoral head for the implanted case, and through the femoral head for the intact case. A planar bearing was mounted between the load cell of the testing machine and the femoral head in order to avoid artificial constraint perpendicular to the loading axis. ${ }^{14,33}$ An initial static load of $100 \mathrm{~N}$ was applied, which was ramped up to $700 \mathrm{~N}$ in $100 \mathrm{~N}$ increments with a constant displacement rate of $10 \mathrm{~mm} / \mathrm{min}$ and a data acquisition rate (Bluehill ${ }^{\circledR}$ software) of $100 \mathrm{~Hz}$. The average allowed relaxation time between two successive load steps were 30 seconds and that between the load step and image acquisition was 20 seconds. The experimental set-up for DIC measurement is shown in Fig. 2 a and b. The anatomical reference system for the analogue femurs is presented in Fig. 3, with the origin located at the base of the machine.

The undeformed and deformed speckle patterns were photographed using 2 MP dual digital cameras (Limess Messtechnik und Software $\mathrm{GmbH}$, Krefeld, Germany), before and after application of the static force, respectively (Fig. 2a). The cameras were placed at an angle of \pm 10 degrees to the normal direction of the region of interest. The camera position relative to the specimen was kept unaltered both for the implanted and the intact cases, in order to have one-to-one comparison of the measured data. From five successive unloaded datasets (theoretically with zero strain), the mean von Mises strain sensitivity was calculated to be $45 \mu \varepsilon$ with $\mathrm{SD} \pm 12 \mu \varepsilon$. A diffused white light source was used for illumination enabling short exposure (12ms) image capture, in order to minimise noise and reflection.

VIC-3D 2010 software (Correlated Solutions Inc., USA) was used for calculation of the strain fields by postprocessing the recorded data. The quantitative differences in von Mises strains between the intact and the implanted femurs were analysed in seven defined Gruen zones in order to quantitatively assess surface strain shielding. A custom MATLAB (vR2011b, The Mathworks, Inc., Natick, MA, USA) code was used to identify the Gruen zones on the analogue femur by specifying landmarks (boundary points) on the recorded images. These polygonal regions, defined by the boundary points, were chosen in such manner that the full-field strain data were free from the noise readings, which are generally associated with regions with high curvature gradients. Thereafter, comparisons of strains between intact and implanted cases were performed by applying rigid registration of the coordinates. 

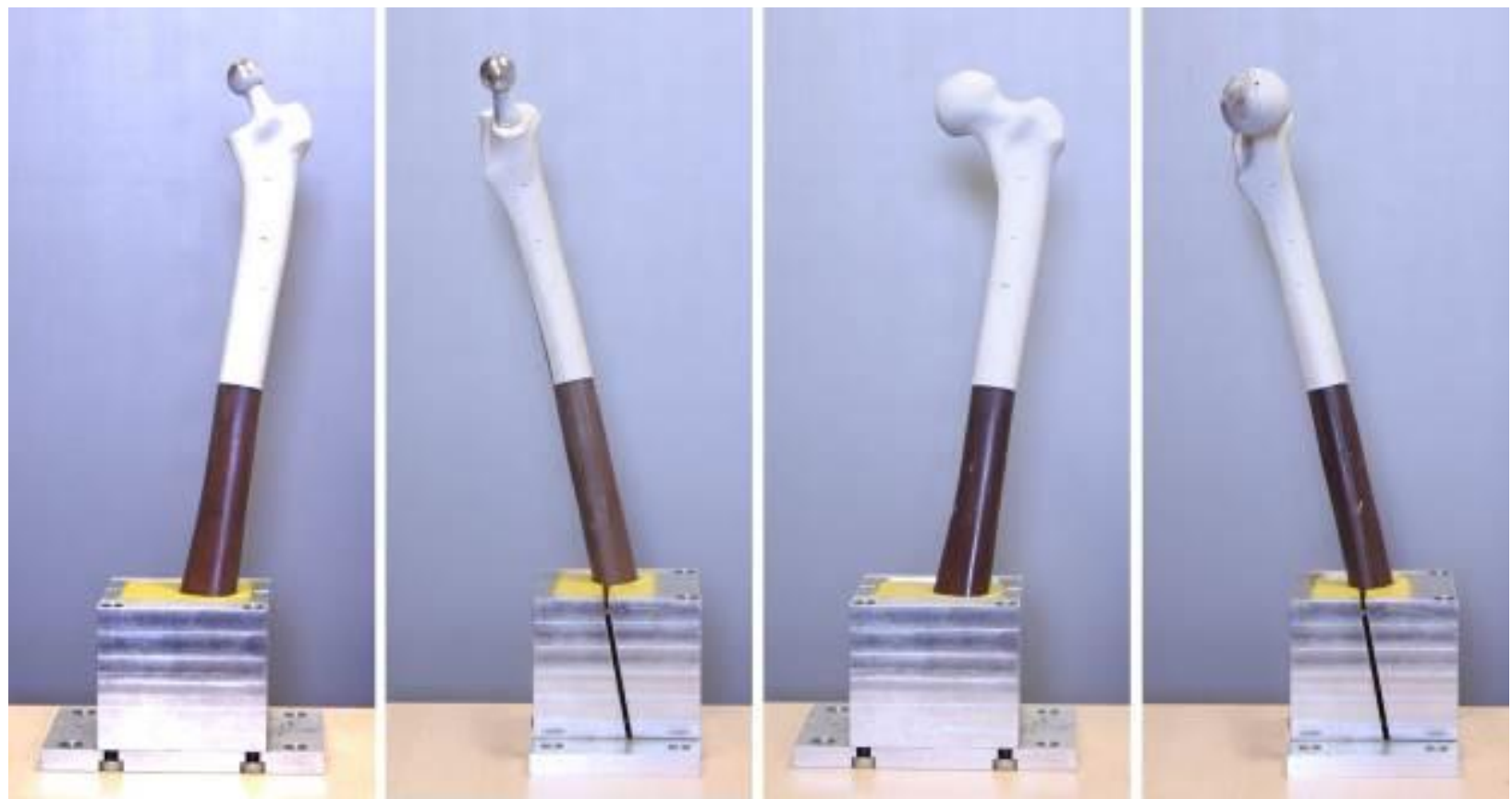

FIGURE 1: The position and orientation of the tested femur specimens: tilted $10^{\circ}$ laterally in the frontal plane for both (a) implanted and (c) intact femur; dorsally by $10^{\circ}$ in the sagittal plane for both (b) implanted and (d) intact femur specimens.

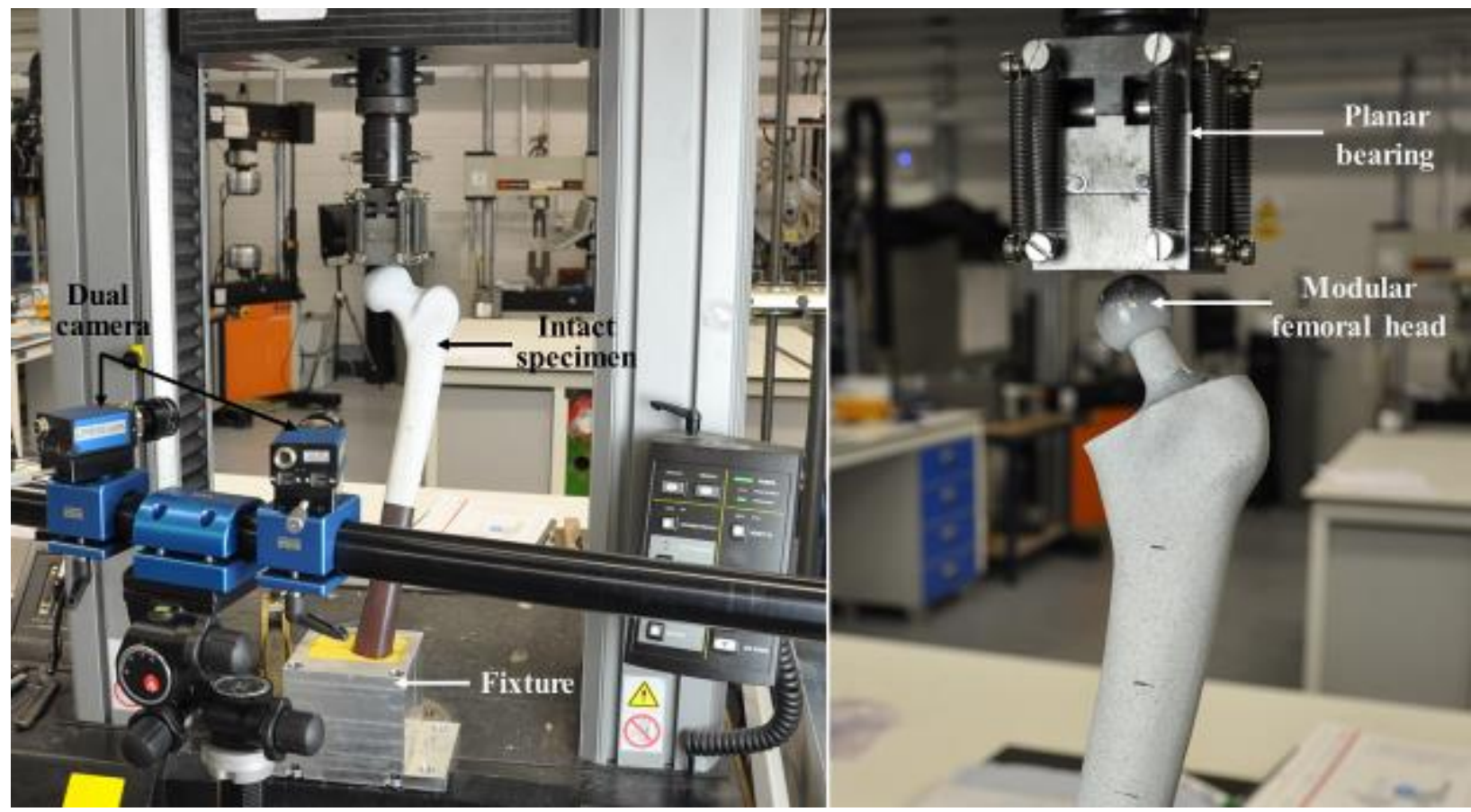

FIGURE 2: The experimental set-up for DIC measurement along with the planar bearing: (left) intact; (right) implanted.

\section{Finite element modelling and analysis}

The 3D FE models of intact and implanted femurs were generated using the manufacturer supplied CAD model of the composite femur. A solid model of the Furlong HAC implant (size $12 \mathrm{~mm}$ ) was reproduced in SolidWorks ${ }^{\circledR}$ software (DS SolidWorks Corp., Concord, MA, USA), as shown in Fig. 3b. The solid models of the femur and the implant were converted into freeform surfaces (.STL format) and imported into Rhinoceros ${ }^{\circledR}$ (Rhinoceros v4.0, Robert McNeel \& Associates, Seattle, USA). The experimental orientation of the composite bone, and the relative position and orientation of the implant and osteotomies, were identified in orthogonal photographs of the experimental setup and reproduced in the CAD model. The composite bone was resected and virtual implantation 
was performed through Boolean operations after positioning the implant in an orientation that replicated the tested specimen (Fig. 3a). The reverse-engineered and triangulated surface models were then imported into ANSYS ICEM CFD (ANSYS Inc., PA, USA) to generate a volumetric mesh comprising 4-noded unstructured tetrahedral elements with maximum edge lengths between $1-3 \mathrm{~mm}$. Thereafter, the volumetric mesh was imported into ANSYS 14 (ANSYS Inc., PA, USA) and converted into ten node tetrahedra before analysis. A mesh size study was carried out to check the numerical convergence of the model using three different FE meshes containing $\sim 614,000, \sim 977,000$ and $\sim 2,662,000$ elements respectively, and a comparison of their predicted surface strain. The maximum difference in peak von Mises strain was predicted to be $8 \%$ between the coarse and the medium meshes, and $2 \%$ between the medium and fine meshes. Therefore, considering the computational efficiency and accuracy, the medium sized mesh was judged to be sufficiently accurate. The implanted FE model used for the study is shown in Fig. 3a. A similar technique was adopted for generating the FE model of the intact femur.

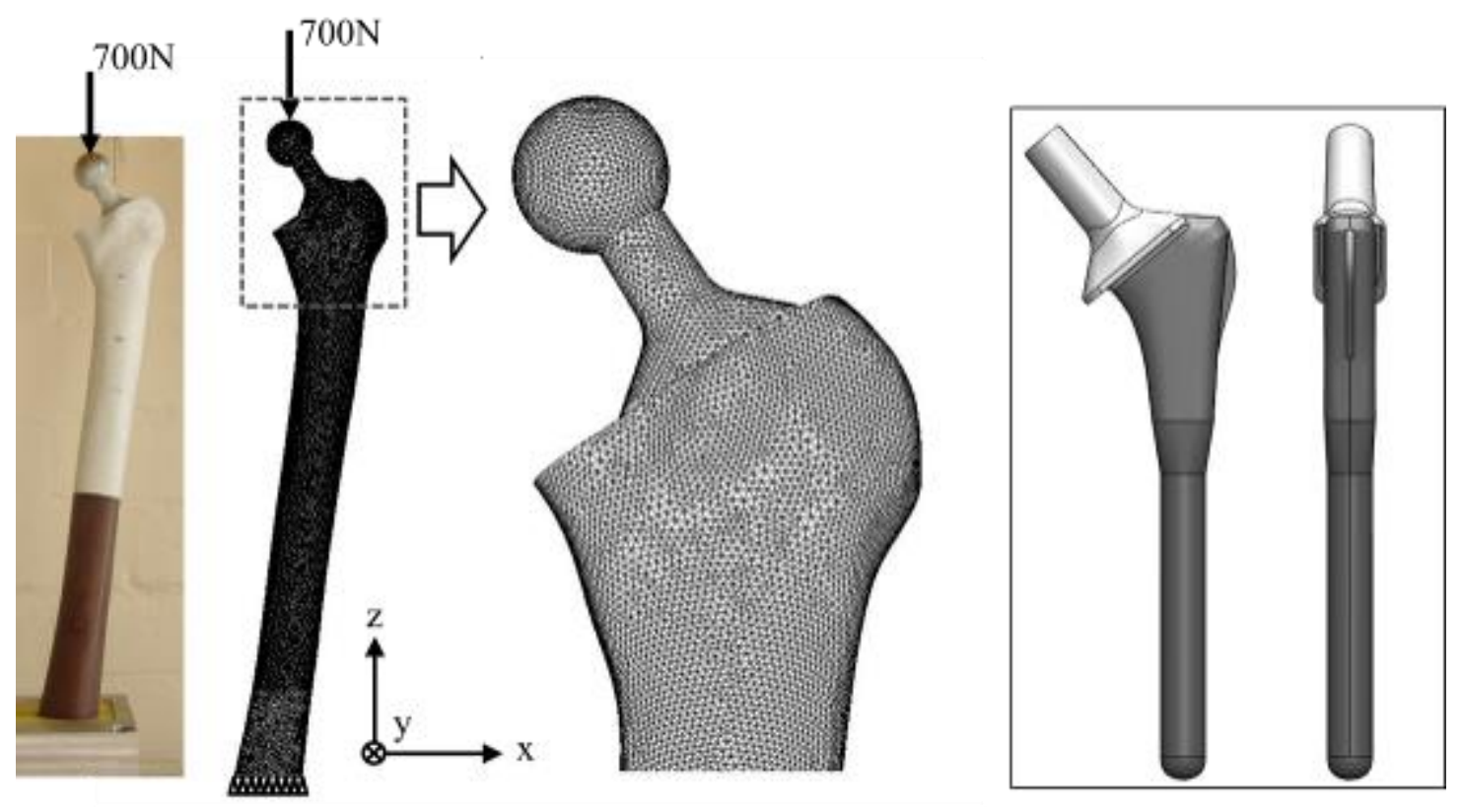

FIGURE 3: Development of FE model for implanted femur: (a) the implanted FE model having identical orientation with respect to the tested specimen, (b) the generic CAD model of a Furlong stem (size $12 \mathrm{~mm}$ ) used for FE modelling.

Based on the data provided by the manufacturer, linear elastic, isotropic and homogeneous material properties were applied to the composite bone, with the cancellous foam and the cortical shell having Young's moduli $E=$ 16.7 $\mathrm{GPa}$ and $E=155 \mathrm{MPa}$, respectively. ${ }^{34}$ The Young's moduli of the Furlong HAC stem and the femoral head were set as $110 \mathrm{GPa}$ (titanium alloy, Ti-6Al-4V) and $210 \mathrm{GPa}$ (cobalt-chromium alloy, CoCr), respectively and the Poisson's ratio for all materials was set as 0.3 . Since a relatively low magnitude of load (700N) was used during the experiment, bonded contact was considered at the implant-bone interface. ${ }^{35}$ Enclosed polygonal regions of the proximal-frontal and proximal-medial parts of the implanted FE model were considered as two Regions of Interest (ROI), ROI-1 and ROI-2, respectively, for verification purposes. Load was applied through the modular femoral head for the implanted model and through the femoral head for the intact model, replicating the experimental condition. Constraints were applied at distal femoral surface nodes at a distance that again replicated the experimental condition.

\section{Data interpretation and statistical analysis}

The full-field DIC strain measurements ('von Mises' or 'equivalent' strain) were compared with the FE predicted results to verify the measurement technique. The linear regression analysis was based on nodal values averaged over $4 \mathrm{~mm} \times 4 \mathrm{~mm}$ virtual strain gauge areas of DIC measured and FE predicted strains obtained from the two 
ROIs, corresponding to 100 points. A Student's t-test was conducted to verify the degree of correlation between the FE predicted and the DIC measured data. The correlation coefficient (R), standard error of estimate (SE), percentage error (PE) and 'test statistics' value (t-statistic) of the regression slope (b) were used to evaluate whether a significant relationship existed between the DIC measured and FE predicted strain, assuming the data points were normally distributed (95\% confidence interval). The percentage error was calculated as the ratio of 'SE of b' to the slope of the regression line (b).

\section{Results}

\section{Comparison of DIC measured strains between intact and implanted femurs}

The von Mises strain distributions for the two ROIs of the implanted and intact proximal femurs are shown in Figures 4 and 5, respectively. The distribution of DIC measured and FE predicted strains for the implanted femur varied from 0 to $370 \mu \varepsilon$ within the two ROIs (Fig. 4). Low strains ( 0 to $125 \mu \varepsilon$ ) were observed in the medial calcar region in the implanted case, which indicated a marked reduction in load transfer in comparison to the intact case $(375-625 \mu \varepsilon)$. The anterior lateral portion demonstrated a similar change, from $125-500 \mu \varepsilon$ (intact) to $0-125 \mu \varepsilon$ (implanted).

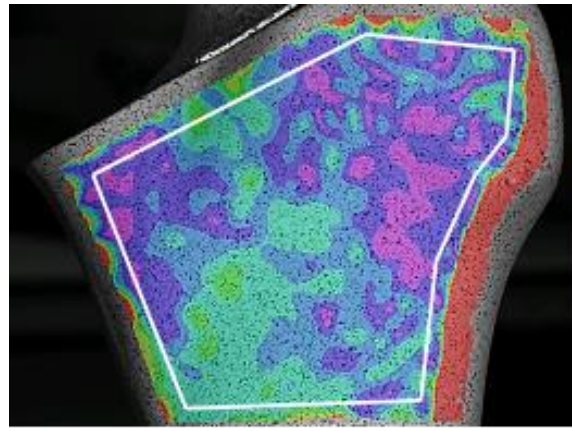

(a)

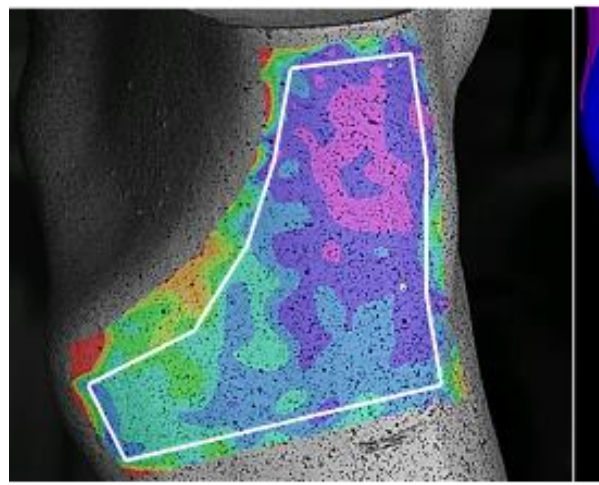

(c)

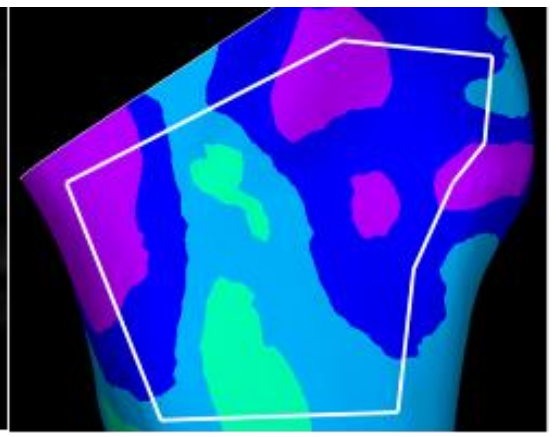

(b)

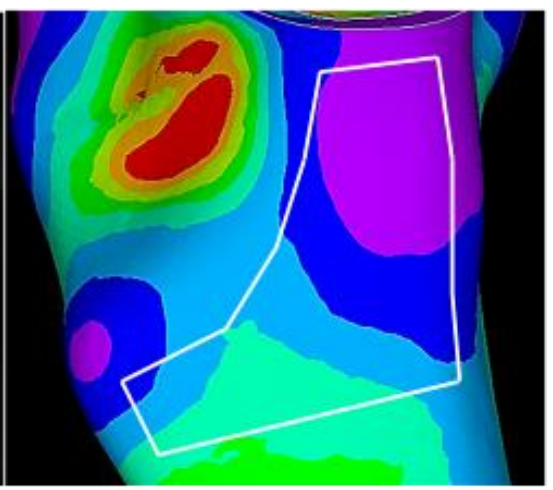

(d)

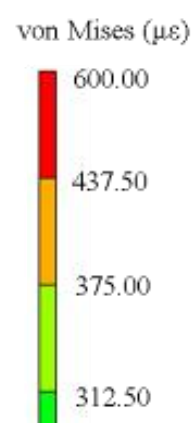

250.00

187.50

125.00

62.50

$\square_{0}$

FIGURE 4: Comparison of DIC vs FE predicted strain in implanted femur: (a), (c) DIC measured strain; (b), (d) FE predicted strain at proximal-frontal (ROI-1) and proximal-medial (ROI-2) femur, respectively. 


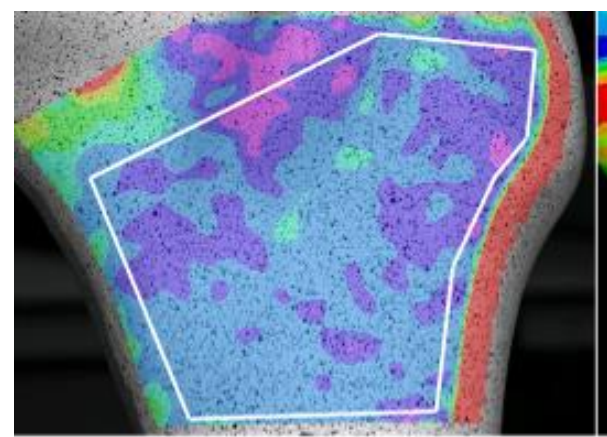

(a)

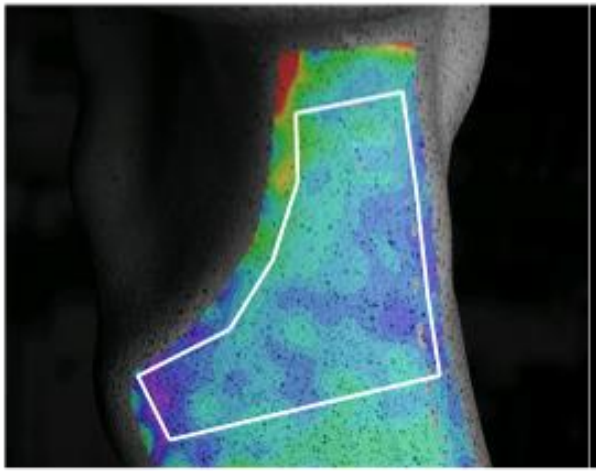

(c)

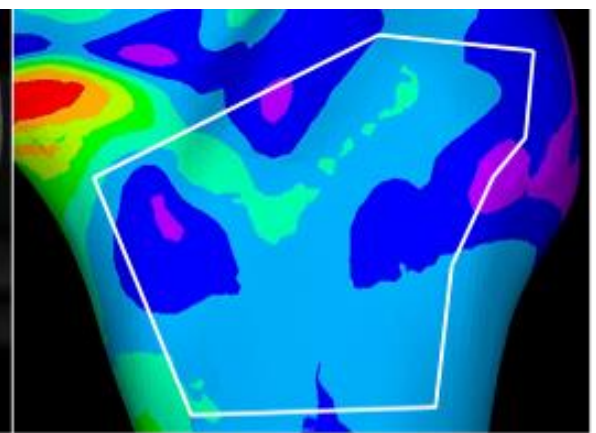

(b)

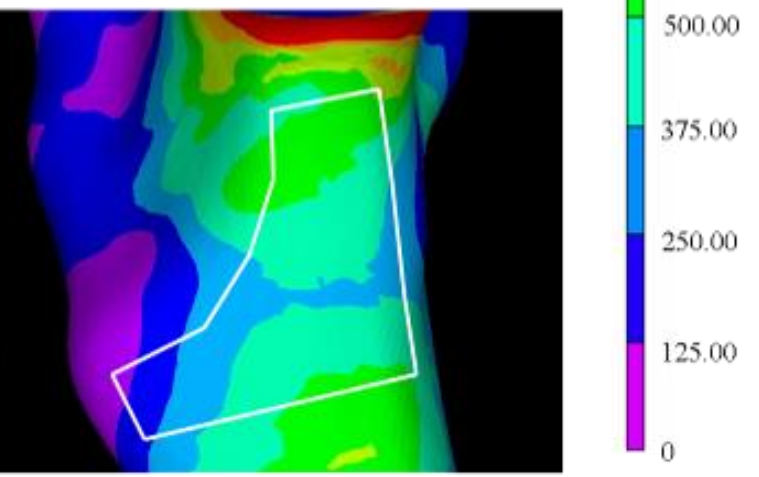

(d)

FIGURE 5: Comparison of DIC vs FE predicted strain in intact femur: (a), (c) DIC measured strain; (b), (d) FE predicted strain at proximal-anterior (ROI-1) and proximal-medial (ROI-2) femur, respectively.

\section{Assessment of the relationship between the DIC measured and FE predicted strains}

The results of the regression analysis are presented in Table 1 and the scatter plots are shown in Fig. 6a and b. Correlations were statistically significant in both implanted and intact cases and the regression slopes were close to unity in both cases (Table 1). The regression slopes for implanted ( $b=0.876, \mathrm{PE}$ of $\mathrm{b}=4.1 \%$ ) and intact $(\mathrm{b}=$ 0.862 , PE of $\mathrm{b}=4.0 \%)$ cases along with the intercepts $(\mathrm{a}=19.78 \mu \varepsilon$ for implanted and $\mathrm{a}=14.88 \mu \varepsilon$ for intact $)$ were indicative of a strong linear relationship between the DIC measured and FE predicted von Mises strains (95\% confidence interval). However, deviations in DIC measured and FE predicted strains (Fig. 6a,b) were primarily observed in the curved regions of the medial ROI, i.e. ROI- 2.

\begin{tabular}{|c|c|c|c|c|c|c|c|}
\hline $\begin{array}{c}\text { Femur } \\
\text { specimen }\end{array}$ & $\begin{array}{c}\text { Correlation } \\
\text { coefficient }(\mathrm{R})\end{array}$ & $\begin{array}{l}\text { Standard error } \\
\text { of estimate } \\
\text { (SE) in } \mu \varepsilon\end{array}$ & $\begin{array}{r}\text { Lin } \\
\text { regres } \\
y=a \\
a(\mu \varepsilon)\end{array}$ & $\begin{array}{l}\text { ir } \\
\text { ion: } \\
b x\end{array}$ & $\begin{array}{l}\text { Standard error } \\
\text { (SE) of } b\end{array}$ & $\begin{array}{c}\% \text { error } \\
(\mathrm{PE}) \text { of } \\
b\end{array}$ & $\begin{array}{l}\mathrm{t} \text {-statistic } \\
\text { ( } \mathrm{p} \text { value) }\end{array}$ \\
\hline $\begin{array}{c}\text { Intact } \\
(\mathrm{N}=100)\end{array}$ & 0.927 & 38.38 & 14.886 & 0.862 & 0.035 & 4.06 & $\begin{array}{l}24.296 \\
(0.000)\end{array}$ \\
\hline $\begin{array}{l}\text { Implanted } \\
(\mathrm{N}=100)\end{array}$ & 0.926 & 26.07 & 19.782 & 0.876 & 0.036 & 4.11 & $\begin{array}{l}24.362 \\
(0.000)\end{array}$ \\
\hline $\begin{array}{c}\text { All data } \\
(\mathrm{N}=200)\end{array}$ & 0.937 & 32.82 & 20.574 & 0.850 & 0.023 & 2.71 & $\begin{array}{l}37.649 \\
(0.000)\end{array}$ \\
\hline
\end{tabular}

TABLE 1: Linear regression analysis of the FE predicted and DIC measured von Mises strain ( $\mu \varepsilon)$ for intact and implanted femur for load $700 \mathrm{~N}$. The number of data points collected from the ROIs is denoted by ' $\mathrm{N}$ '. 

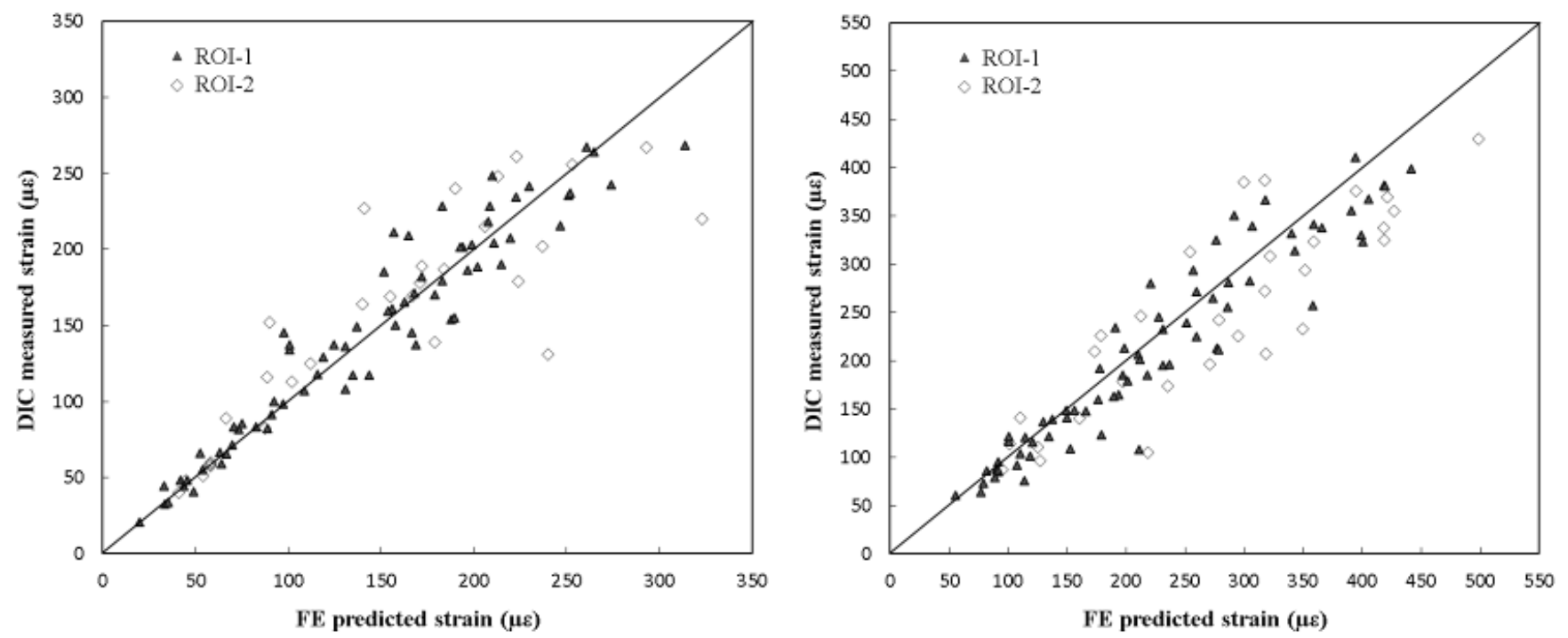

FIGURE 6: Measured strain plotted against FE predicted strain obtained from the two ROIs (ROI-1 and ROI-2), with respect to the ideal line (slope $=1$, intercept $=0$ ), for $700 \mathrm{~N}$ load in $(\mathrm{a})$ implanted and $(\mathrm{b})$ intact femur specimens.

\section{Strain shielding in implanted femur across seven Gruen zones}

The von Mises strain distributions in Gruen zones both for the intact and implanted femur, along with a bar chart illustrating percentage change of strain in each zone, are shown in Fig. 7. At $700 \mathrm{~N}$ load, the measured strain values in GZ-4 ranged between $100-750 \mu \varepsilon$ for the implanted case and $20-470 \mu \varepsilon$ in the intact femur. However, in GZ-1 and -7 of the implanted femur, very low strains (mean $\sim 130 \mu \varepsilon, \mathrm{SD} \pm 70 \mu \varepsilon$ ) were observed, whereas at comparable locations in the intact femur, the strain values were found to be higher (mean $\sim 300 \mu \varepsilon, \mathrm{SD} \pm 85 \mu \varepsilon$ ).

The mean reductions in measured and FE predicted equivalent strain in the implanted femur across each of the seven Gruen zones compared to the intact case are shown in Figures 7a and 7d, respectively. Considerable reductions in measured strain were observed in the lateral and medial regions of the implanted femur; GZ-1 recorded $\sim 56 \%$ reduction in mean strain and GZ-7 demonstrated $\sim 50 \%$ reduction with a standard deviation of $\sim \pm 7 \%$ for both zones (Fig. 7a). 


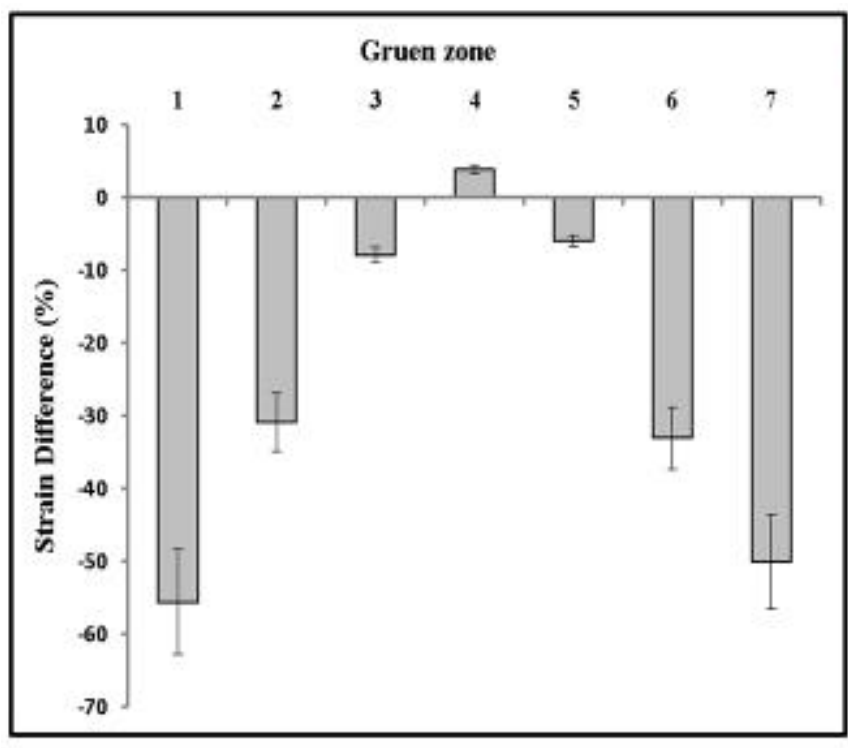

(a)

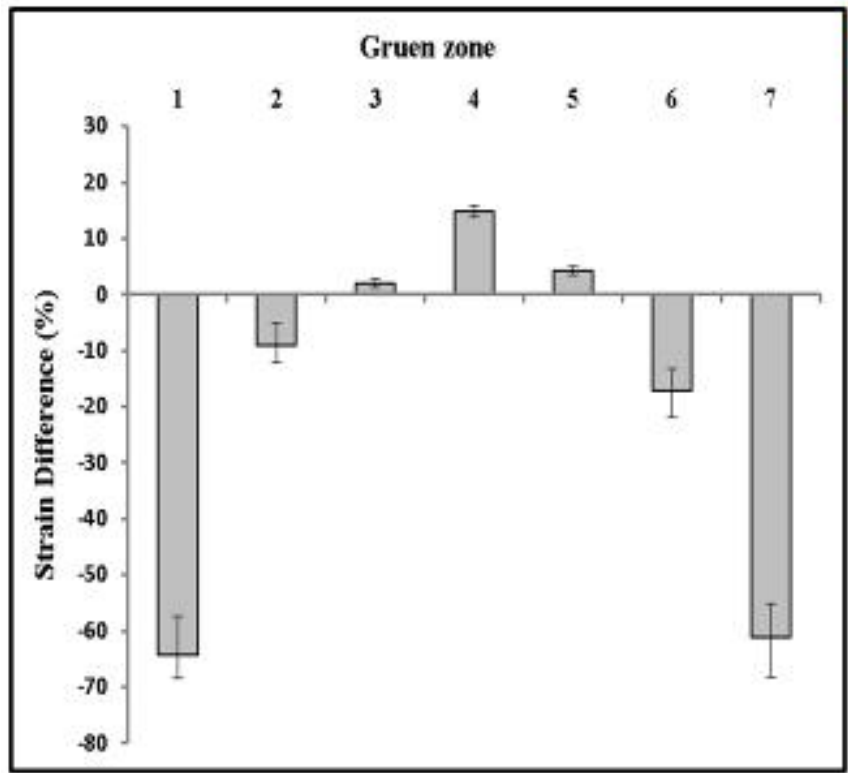

(d)

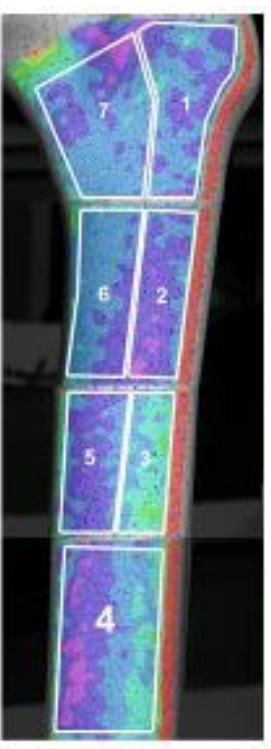

(b)

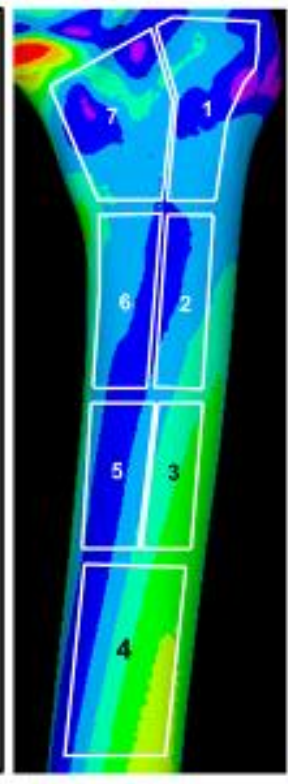

(e)

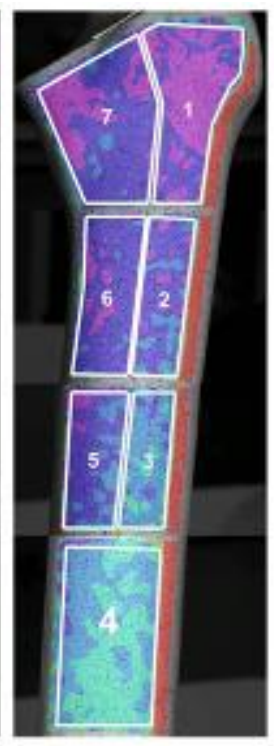

von Mises ( $u \varepsilon$ )

1000.00

875.00

750.00

625.00

(c)

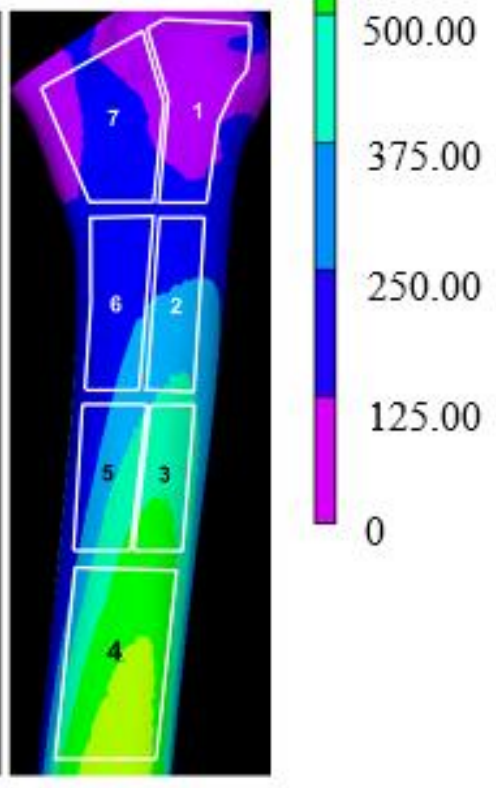

(f)

FIGURE 7: Changes in strain distribution due to implantation: (a) DIC measured and (d) FE predicted percentage difference in von Mises strains in Gruen zones (GZ 1-7) between the implanted and intact femurs. Strain distribution in intact femur (GZ 1-7) for (b) measured and (e) predicted and strain distribution in implanted femur (GZ 1-7) for (c) measured and (f) predicted strain, respectively. 


\section{Discussion}

The first objective of this study was to verify the FE models of intact and implanted femurs using DIC. This was necessary to support the second phase of the work where these models were used for quantitative assessment of implant-induced surface strain shielding. Full-field surface strain comparisons were carried out between the intact and the implanted bone across seven Gruen zones. For the purpose of one-to-one comparison, von Mises (equivalent) strain on the bone surface was chosen. When an object is subject to a set of multi-axial loads, the von Mises strain, which takes into account the effect of each strain component, can be used as a magnitude-based measure for comparison between DIC measured and FE predicted strains provided the object deformation is predominantly in-plane. Furthermore, the derivatives of the out-of-plane displacement are significantly less than those of the in-plane displacement, so that these effects may be excluded from analysis. In the present FE analysis, the out-of-plane strain components were found to be negligibly small compared to the in-plane components in the ROIs. However, this assumption may not be valid in regions with highly curved boundaries, leading to deviations in DIC measured and FE predicted strains. Hence, these regions were excluded from the analysis.

Full-field strain distributions for both the specimens were found to be very similar to the FE predicted strain patterns. However, some deviations, especially at the curved areas of the ROIs, may be attributed to the subtended acute angle in the viewing direction of the cameras. To improve accuracy, close-up data acquisition at strain peak regions with cameras positioned closer to the normal of the surface may be attempted. Since the resolution depends on the pixel subset size used for strain calculation, DIC (to a lesser extent than strain gauging) could also underestimate the peak strain due to averaging errors, especially at the curved boundaries of the ROIs. This is a limitation of the technique. Therefore, a finer speckle pattern combined with a closely positioned camera may result in greater measurement sensitivity. The FE prediction may be overestimated through a loss in thickness of the cortex structure in the model, arising from CAD modelling or FE meshing. However, a close linear agreement obtained with the full-field strain data gave confidence in the validity of the present model. Linear regression analysis indicated close agreement between the DIC measured and FE predicted strain, with a high correlation coefficient $(\mathrm{R}=0.92$ - 0.94), low SE of the estimate $(26-38 \mu \varepsilon)$, and low PE in the regression slope $(\sim 2-4 \%)$. These results indicate that the overall measured data corroborated well with the FE predicted data. The results are also consistent with the findings of some recently published studies. ${ }^{14,19}$ Dickinson et al. ${ }^{14}$ reported correlation coefficient in the range $0.91-0.96$ between the test and simulation results, with slope lying within $0.90-0.93$ and intercept within $44-90 \mu \varepsilon$. Grassi et al. ${ }^{19}$ reported a correlation coefficient of $\sim 0.93$ with an SE value of $915 \mu \varepsilon$ (7\%) and slope and intercept values were found to be 0.84 and $128 \mu \varepsilon$, respectively.

The second objective of the present research was to quantitatively assess the post-implantation strain shielding across Gruen zones, which may be indicative of clinically observed bone density changes around similar implants. It can be seen from the load transfer pattern of the implanted femur (Fig. 7a,d) that GZ-1 and GZ-7 experienced maximum strain shielding due to implantation and these are the regions of maximum observed implant-induced long term bone resorption clinically. ${ }^{1,2,36,37}$ On the other hand, GZ-4 demonstrated a slight increase in strain values ( $\sim \%$ measured; $\sim 14 \%$ predicted), indicating a propensity for longer term bone apposition. Rosenthall et al. ${ }^{38}$, in a 4-year prospective radiographic study of bone densitometry with hydroxyapatite-coated implants, reported that all Gruen zones had registered significant BMD loss at 6 months. However, Gruen zones 4 and 5 exhibited maximum mineral recovery $(\sim 1-5 \%)$ after 4 post-operative years. The early drop in BMD in the short postoperative term is likely to be related to surgically introduced microdamage and reduced immediate postoperative activity and joint 
loading, ${ }^{39}$ and the osteoclastic remodelling transient which occurs prior to osteoblastic bone densification, which is measurable following THA. ${ }^{40}$ In an extended follow-up study (mean duration of follow-up 22.5 years) with hydroxyapatite-coated (HAC) Furlong stems, Sandiford et $a .^{41}$ reported localized bone resorption immediately under the collar (Gruen zone 7). While Gruen zones 2 and 3 showed signs of radiolucency in the study, they were found to be non-progressive. Calcar atrophy was also noted in other follow-up studies for the Furlong HAC stem, indicating resorption in Gruen zone 7. ${ }^{42,43}$ Moreover, Singh et al. ${ }^{43}$ noted formation of new bone extending from the femoral cortex to the stem-tip, i.e. in Gruen zone 4, consistent with the slight increase in strain at GZ-4 observed in this study.

The present study, however, has certain limitations; the primary among them is the loading condition. The test loading and boundary conditions are not entirely representative of the in vivo condition, where there are no rigid constraints. In addition, only one representative loading condition within a walking cycle was considered on a single analogue specimen and no muscle forces were introduced into the study. These limitations may be justified in the present scenario, the proximal femur implanted with a long-stemmed THA, as indicated by the correlations observed between the surface strain measurements and clinical BMD data. Other arthroplasty types and joints may however require more detailed and complex representations of the true musculoskeletal loading and support conditions. Moreover, although a moderate load of $700 \mathrm{~N}$ was used into the study, it could be noted from the results that the loading was sufficient to achieve an acceptable signal to noise ratio. However, such moderate load may introduce further assumption of linear material behaviour versus load. The use of composite bone, however, may be justified because it allows reliability and repeatability of the DIC measurements. Furthermore, the interface condition used in the implanted FE model may differ from the actual interface conditions existing in the implanted femur. Some of the discrepancies between measured and predicted strains may also have arisen from the linear isotropic modelling of bone properties in the FE models. Moreover, it may be argued that the use of cortical surface strains may not be entirely appropriate, as they are not indicative of the internal trabecular deformations. However, the verification of surface strain by Keyak et al. ${ }^{44}$ supported the validity of the internal strain and stress predictions in the proximal femur, because surface strain is intimately related to strain inside the bone. ${ }^{45}$ In the present study conventional Gruen zones were used; however, there is a justification for higher resolution assessment of DIC and clinical DXA data over smaller regions, such as the $4 \mathrm{~mm} \times 4 \mathrm{~mm}$ virtual strain gauge zones used in this study. Gruen zones have been adopted for assessment of periprosthetic bone adaptation, but in vitro (FE, DIC) and in vivo (DXA) techniques offer more information through their higher spatial resolution. This practice is becoming established in clinical research, where DXA data is interrogated in a greater number of smaller regions. ${ }^{46}$ Ultimately, further experimentation, with high spatial resolution strain analysis, on multiple cadaver specimens using complex loading conditions would be more useful to gain an insight into the relationship between strain shielding and long-term postoperative bone density changes.

This study, however, provides a technique by which researchers can evaluate the relative extents to which bone strain is influenced by different implants, and may predict part of the mechanical contribution to periprosthetic bone density changes after joint replacement. The significance in correlation between measured and predicted surface strain in a cementless total hip replacement supports the use of an FE model as a valid predictor of the measured strain. The changes in both measured and FE predicted surface strain distribution due to implantation were found to correlate well with clinical trends in volumetric BMD changes; marked resorption is seen in GZs 1, 6 and 7 which correlated well with measured reduction in surface strain, and slight density reduction or increase is seen in GZ-3, -4 and -5 , which corroborated with the measured and predicted increase in 
strain. The present research therefore also supports the use of DIC as a pre-clinical analysis tool for prediction of adaptive bone density changes through measurement of post-implantation strain states.

\section{Acknowledgements}

The authors would like to thank the UKIERI British Council for supporting this study. The authors would also like to acknowledge JRI Orthopaedics for providing the implant model and Mr. Andrew Jones, Consultant Orthopaedic Surgeon, Great Western Hospitals NHS Foundation Trust for performing the implantation.

\section{Conflict of interest}

The authors hereby declare that with regard to the submission of this article there are no financial or personal relationships with other people and organisations.

\section{References}

1. Engh CA, Bobyn JD and Glassman AH. Porous-coated hip replacement. the factors governing bone ingrowth, stress shielding, and clinical results. J Bone Jt Surg Br 1987; 69: 45-55.

2. Engh CA and Bobyn JD. The influence of stem size and extent of porous coating on femoral bone resorption after primary cementless hip arthroplasty. Clin Orthop 1988; 231: 7-28.

3. Evola FR, Evola G, Graceffa A, et al. Performance of the CLS Spotorno uncemented stem in the third decade after implantation. Bone Jt J 2014; 96-B: 455-461.

4. Kerner J, Huiskes R, van Lenthe GH, et al. Correlation between pre-operative periprosthetic bone density and post-operative bone loss in THA can be explained by strain-adaptive remodelling. J Biomech 1999; 32: 695-703.

5. Nishii T, Sugano N, Masuhara K, et al. Longitudinal evaluation of time related bone remodeling after cementless total hip arthroplasty. Clin Orthop 1997; 339: 121-131.

6. Bagge M. A model of bone adaptation as an optimization process. J Biomech 2000; 33: 1349-1357.

7. Huiskes R, Weinans $H$ and van Rietbergen $B$. The relationship between stress shielding and bone resorption around total hip stems and the effect of flexible materials. Clin Orthop Rel Res 1992; 274: 124-134.

8. Ruben RB, Folgado J and Fernandes PR. On the optimal shape of hip implants. J Biomech 2012; 45: $239-246$.

9. ten Broeke RHM, Tarala M, Arts JJ, et al. Improving peri-prosthetic bone adaptation around cementless hipstems: a clinical and finite element study. Med Eng Phys 2014; 36: 345-353.

10. Decking R, Puhl W, Simon U, et al. Changes in strain distribution of loaded proximal femora caused by different types of cementless femoral stems. Clin Biomech 2006; 21: 495-501.

11. Gillies RM, Morberg PH, Bruce WJ, et al. The influence of design parameters on cortical strain distribution of a cementle ss titanium femoral stem. Med Eng Phys 2002; 24: 109-114.

12. Gronewold J, Berner S, Olender G, et al. Changes in strain patterns after implantation of a short stem with metaphyseal ancho rage compared to a standard stem: an experimental study in synthetic bone. Orthop Rev (Pavia) 2014; 6: 5211.

13. Kim YH, Kim JS and Cho SH. Strain distribution in the proximal human femur. An in vitro comparison in the intact femur and after insertion of reference and experimental femoral stems. J Bone Jt Surg 2001; 83: 295-301.

14. Dickinson AS, Taylor AC and Ozturk HMB. Experimental validation of a finite element model of the proximal femur using digital image correlation and a composite bone model. Trans ASME J Biomech Eng 2011; 133: 1-6.

15. Sztefek P, Vanleene M, Olsson R, et al. Using digital image correlation to determine bone surface strains during loading and after adaptation of the mouse tibia. J Biomech 2010; 43: 599-605.

16. Väänänen SP, Amin Yavari S, Weinans H, et al. Repeatability of digital image correlation for measurement of surface strains in composite long bones. J Biomech 2013; 46: 1928-1932.

17. Cofaru C, Philips W and van Paepegem W. Pixel-level robust digital image correlation. Optics Exp 2013; 21: 29979-29999.

18. Kahn-Jetter ZL and Chu TC. Three-dimensional displacement measurements using digital image correlation and photogrammic analysis. Exp Mech 1990; 30: 10-16. 
19. Grassi L, Väänänen SP, Amin Yavari S, et al. Experimental validation of finite element model for proximal composite femur using optical measurements. J Mech Behav Biomed Mat 2013; 21: 86-94.

20. Ajovalasit A, Fragapane S and Zuccarello B. Local reinforcement effect of embedded strain gauges. EPJ Web of Conferences 2010, 6.

21. Beatty MF and Chewning SW. Numerical analysis of the reinforcement effect of a strain gage applied to a soft material. Int $J$ Eng Sci 1979; 17: 907-915.

22. Perry CC. Strain-gage reinforcement effects on orthotropic materials. Exp Tech 1986; 10: 20-24.

23. Chuang SF, Chang $\mathrm{CH}$ and Chen TYF. Contraction behaviors of dental composite restorations - finite element investigation with DIC validation. J Mech Behav Biomed Mat 2011; 4: 2138-2149.

24. Moerman KM, Holt C, Evans SL, et al. Digital image correlation and finite element modeling as a method to determine mechanical properties of human soft tissue in-vivo. J Biomech 2009; 42: 1150-1153.

25. Op Den Buijs J and Dragomir-Daescu D. Validated finite element models of the proximal femur using two-dimensional projected geometry and bone density. Comp Meth Prog Biomed 2011; 104: 168-174.

26. Tayton E, Evans S and O'Doherty D. Mapping the strain distribution on the proximal femur with titanium and flexible-stemmed implants using digital image correlation. J Bone Jt Surg Br 2010; 92-B: 1176-1181.

27. Ozturk H, Multi parameter computational and experimental investigations into the robustness of cementless total hip replaceme nts, PhD Thesis, May 2011, University of Southampton, Southampton, UK.

28. Gilchrist S, Guy P and Cripton PA. Development of an inertia-driven model of sideways fall for detailed study of femur fracture mechanics. Trans ASME J Biomech Eng 2013; 135: doi: 10.1115/1.4025390.

29. Grassi L, Väänänen SP, Yavari SA, et al. Full-field Strain Measurement During Mechanical Testing of the Human Femur at Physiologically Relevant Strain Rates. Trans ASME J Biomech Eng 2014; 136: doi: 10.1115/1.4028415.

30. Helgason B, Gilchrist S, Ariza O, et al. Development of a balanced experimental-computational approach to understanding the mechanics of proximal femur fractures. Med Eng Phys 2014; 36: 793-799.

31. Dickinson AS, Taylor AC and Browne M. The influence of acetabular cup material on pelvis cortex surface strains, measured using digital image correlation. J Biomech 2012; 45: 719-723.

32. ISO 7206-4:2002 Implants for surgery - Partial and total hip joint prostheses - Part 4: Determination of endurance properties of stemmed femoral components.

33. Pal B, Gupta S, New AM, et al. Strain and micromotion in intact and resurfaced composite femurs: experimental and numerical investigations. J Biomech 2010; 43: 1923-1930.

34. http://www.sawbones.com

35. Bougherara H, Zdero R, Shah S, et al. A biomechanical assessment of modular and monoblock revision hip implants using FE analysis and strain gage measurements. J Orthop Surg Res 2010; 5: 34.

36. Rahmy AI, Gosens T, Blake GM, et al. Periprosthetic bone remodelling of two types of uncemented femoral implant with proximal hydroxyapatite coating: a 3-year follow-up study addressing the influence of prosthesis design and preoperative bone density on periprosthetic bone loss. Osteoporos Int 2004; 15: 281-289.

37. Weeden SH and Paprosky WG. Minimal 11-year follow-up of extensively porous-coated stems in femoral revision total hip arthroplasty. J Arthrop 2002; 17[4 Suppl. 1]: 134-137.

38. Rosenthall L, Bobyn JD and Tanzer M. Bone densitometry: influence of prosthetic design and hydroxyapatite coating on regional adaptive bone remodelling. Int Orthop 1999; 23: 325-329.

39. Dickinson AS. Activity and loading influence the predicted bone remodeling around cemented hip replacements. Trans ASME J Biomech Eng 2014; 136: doi: 10.1115/1.4026256.

40. Wilkinson JM, Eagleton AC, Stockley I, et al. Effect of pamidronate on bone turnover and implant migration after total hip arthroplasty: a randomized trial. J Orthop Res 2005; 23: 1-8.

41. Sandiford N, Doctor C, Rajaratnam SS, et al. Primary total hip replacement with a Furlong fully hydroxyapatite-coated titanium alloy femoral component: Results at a minimum follow-up of 20 years. Bone Jt J 2013; 95-B: 467-471.

42. Shetty AA, Slack R, Tindall A, et al. Results of a hydroxyapatite-coated (Furlong) total hip replacement: a 13- to 15-year follow-up. J Bone Jt Surg Br 2005; 87: 1050-1054.

43. Singh S, Trikha SP and Edge AJ. Hydroxyapatite ceramic coated femoral stems in young patients: a prospective ten-year study. $J$ Bone Jt Surg Br 2004; 86-B: 1118-1123.

44. Keyak JH, Meagher JM, Skinner HB, et al. Automated three-dimensional finite element modelling of bone: a new method. $J$ Biomed Eng 1990; 12: 389-397.

45. Keyak JH, Fourkas MG, Meagher JM, et al. Validation of an automated method of 3-dimensional finite element modelling of bone. J Biomed Eng 1993; 15: 505-509.

46. Scott DF. Femoral bone mineral density loss after short stem neck-sparing, resurfacing, and conventional hip arthroplasty. Bone Jt J 2013; 95-B [Suppl. 34]: 525. 